\title{
Research on three dimensional visualization technologies
}

\author{
Han Wang ${ }^{1, a}$ \\ ${ }^{1}$ College of Information Engineering, Capital Normal University, Beijing 100048, China; \\ aljz1994824@163.com
}

Keywords: three dimensional visualization technologies; Surface model; Solid model; Mixed model

\begin{abstract}
Three dimensional visualization technologies are widely used in the field of medicine and industry. At first, the paper introduces the concept of 3D visualization technology, and analyze the principle and key technology of 3D visualization, that modeling based on surface model, solid model and mixed model. Then the paper introduces the main development tools of 3D visualization. In the end, the development of 3D visualization technology is prospected.
\end{abstract}

\section{Introduction}

3D Visualization technology is a comprehensive leading edge technology which is a collection of computer data processing and the image display in the middle of 1980s [1]. It is an image display tool that use 3D seismic data volume to display, describe and explain the underground geological phenomena and characteristics. It can make the geophysicists and geologists "down to" the data body, and understand the occurrence and development of various geological phenomena and the link between each other more deeply. 3D visualization technology is a means of describing and understanding model, and it is also a characteristic of the data body form, but not a simulation technique. It uses a large amount of data, inspects the continuity of data, identifies the data authenticity, and finds and puts forward useful anomaly, to provide a useful tool for the analysis, understanding and repeated data [2]. It's also a bridge for the collaboration of multidisciplinary communication.

\section{The overview of 3D visualization technology}

3D visualization technology is based on computer science, and now it has penetrated into all disciplines. It's also useful and provide great helps in scientific research on these subjects of geography, resources, environment, surveying and mapping science, oceanography, building science, biomedical disciplines [3]. In construction, transportation, medicine and other fields, for example, the three dimensional visualization technology can improve the predictability of policy makers, and can be an early evaluation of the quality and results, to avoid unnecessary waste and loss; In the application of animation and the virtual world, let's enjoy it brings us strong visual impact and the perfect reappearance of the real world that we can roam ancient castle and the vast space; In the application of simulation technology, it can provide certain decision effect to medical operation implement, machinery manufacturing, mineral mining processing, and the construction of water conservancy facilities. The development of 3D visualization technology, has driven the progress of many disciplines. The research of 3D visualization has very important significance.

\section{The key technology of 3D visualization}

After dealing with the modeling of all kinds of features, to truly show this on a computer screen, it also needs a series of necessary transformation, including data preprocessing, the three dimensional transformation, illumination model, texture mapping, etc., the general process of 3D visualization is shown in figure 1 : 


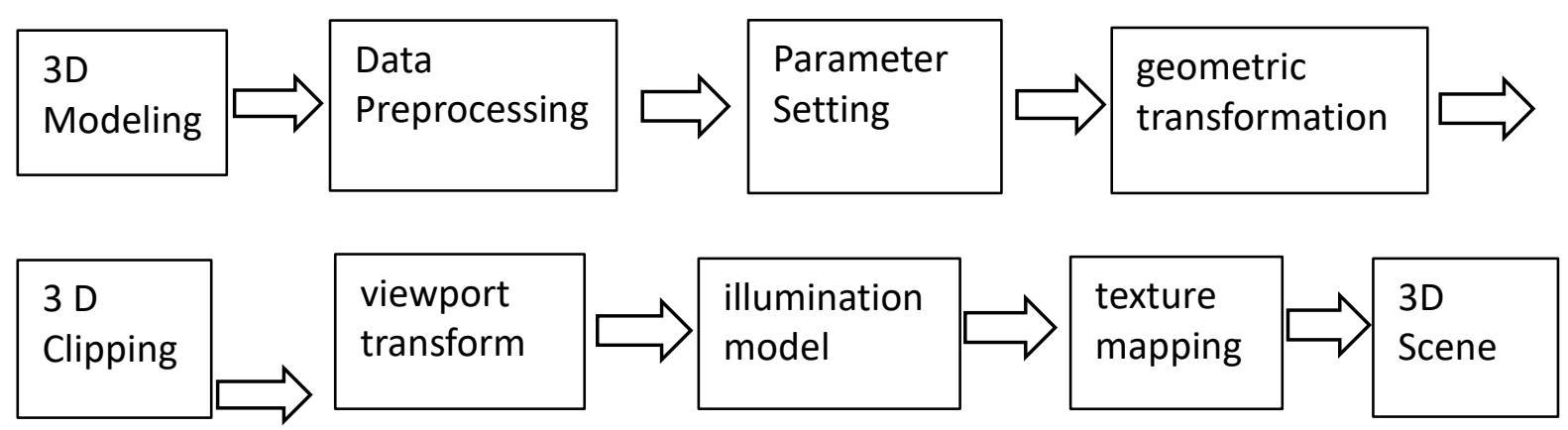

Figure $13 \mathrm{D}$ visualization process

Three-dimensional modeling method research is a research hotspot in the field of 3D visualization of space information. Many experts and scholars have made beneficial exploration in this field. in the past decade, it has been more than 20 kinds of spatial modeling method is proposed. This article uses the plan that put forward by Li-Xin Wu and so on. They reduce space modeling method to Facial Model, Volumetric Model and Mixed Model the three kinds of modeling system (shown in table 1). In the following, we will introduce the basic ideas of the three kinds of technology respectively.

Table 1 3D spatial modeling method of classification

\begin{tabular}{|l|l|l|l|}
\hline \multirow{2}{*}{ Facial Model } & \multicolumn{2}{|c|}{ Volumetric Model } & Mixed Model \\
\cline { 2 - 4 } $\begin{array}{l}\text { Triangular Irregular } \\
\text { Network }\end{array}$ & $\begin{array}{l}\text { Component } \\
\text { Grid }\end{array}$ & Irregular component & $\begin{array}{l}\text { TIN-CSG mixed } \\
\text { model }\end{array}$ \\
\hline $\begin{array}{l}\text { Boundary } \\
\text { Representation }\end{array}$ & Octree & Pelrahedral Network & $\begin{array}{l}\text { TIN-Octree or } \\
\text { Hybrid mixed } \\
\text { model }\end{array}$ \\
\hline $\begin{array}{l}\text { Wire Frame / } \\
\text { Linked slices }\end{array}$ & Needle & Triangular Prism & $\begin{array}{l}\text { Wire Frame-Block } \\
\text { mixed model }\end{array}$ \\
\hline Series Sections & Regular Block & Geocellular & $\begin{array}{l}\text { Octree-TEN mixed } \\
\text { model }\end{array}$ \\
\hline Section-TIN mixed & & Solid & \\
\hline Multilayer DEMs & & 3D Voronoi figure & \\
\hline & & Generalized Tri-prism & \\
\hline
\end{tabular}

\subsection{The modeling method based on Facial Model}

Modeling method based on facial model is focused on the surface of the three-dimensional entity, such as surface topography, geology and structures (building) and the outline of underground engineering and space framework [4]. The simulated surface may be closed, or may be nonocclusive. TNI model based on sampling points and the Grid model based on data interpolation, are typically used for the sealing surface simulation. These two kinds of facial model is commonly used in terrain surface modeling, and they also can be used in the bedded deposit modeling. But B Rep model always to define the location and shape of the form from plane, ring, edge and point; Wire Frame model is usually used in a closed surface or an external contour simulation; Section model, the Section-TIN mixture model and multi-layer DEM model are usually be used in geological modeling. Profile modeling technology are the computer implementation of the traditional geological mapping method, namely through the floor plan or section to describe ore deposit, geological information. Through the surface formed the three dimensional space target outline, its advantage is easy to display and data updates, and its deficiency is due to the lack of three-dimensional geometry description and internal property records, it's difficult to query and analyze three-dimensional spatial. 


\subsection{The modeling method based on Volumetric Model}

Volumetric model based on the three dimensional space element segmentation and true 3D entity, the attributes of voxel can independence describe and store, and thus it can manipulate and analyze three-dimensional space. Volumetric model can be divided into Tetrahedral, Hexahedral, Prismatic and Polyhedral, a total of 4 types, by number of voxel Sides. And it also can be divided into component and Irregular component, two broad categories, according to the regularity of the body. Component including the GSG - tree, Voxel, Octree, Needle and Regular Block, a total of 5 kinds of model. Component is usually used in modeling water, pollution and environmental problems. Irregular component including TEN, Pyramid, TP, Geocelluar, Irregular Block, Solid, 3D - Voronoi and GTP, a total of 8 kinds of model made. Irregular component is a 3D model in the entity which is sampling constraints, and based on the geological formation interface and geological structure.

\subsection{Mixed modeling}

Modeling method based on facial model is focused on the surface of the 3D spatial entity, such as surface topography, geological layer. According to the surface to form a 3D object space. Its advantages are easy to display and data updates, but its deficiencies are difficult to spatial analysis. Modeling method based on Volumetric Model is focuses on the whole show of the boundary of three-dimensional entity with the internal, through the description of the body to achieve the expression of 3D space, and the advantage is easy to space operation and analysis, but the storage space is big while the calculation speed is slow. The purpose of the mixed model is integrated the advantages of facial model and Volumetric Model, and the advantages of component and Irregular component and learn from each other.

\section{3D visualization tools}

The three-dimensional modeling technology is mature, many methods has been integrated into the commercial software. The rapid development of computer graphics technology led the brisk development of 3D modeling technology. There are more and more 3D visualization and graphics rendering tools. The more representative of which are the OpenGL, open international graphics standard of SGI Company, Direct3D of Microsoft, VRML (Virtual Reality Modeling Language) which brings three-dimensional world into network, Java3D, 3D graphics technology based on Java Language of SUN Company, IDL of RSI Company and Vega of Paradigm Company and so on. This software involve the main areas including deposit modeling, design planning, production management, etc. In recent years, with the continuous development of computer technology, some new technologies appear constantly, the emergence of these new technologies provide a new means for $3 \mathrm{D}$ visualization.

\subsection{OpenGL}

OpenGL is an abbreviation for Open Graphics Lib, it is a low-level Graphics library, and it is developed by SGI Company originally. It is a set of 3D graphics library, and also the industry standard in this field.it is a high-performance development package to draw high realistic 3D graphics, interactive visual simulation and virtual display. OpenGL is a process language rather than a descriptive language fundamentally. OpenGL provides the basic operations of direct control of a 2D and 3D geometry. OpenGL offers a wide range of graphics transformation function, when to programme so that don't have to complex matrix operations, and can easily display 3D graphics in the screen window. And, in order to enhance the sense of reality, OpenGL also provides a series of functions that combine blanking, coloring of line and plane, and illumination, texture mapping and anti-aliasing technology. It is helpful to avoid the pure graphics algorithm, simplify the programming, and be very convenient to drawing, coloring and lighting the ground level.

\subsection{Direct3D}

Microsoft DirectX provides a very good application interface, which contains the design of high performance and real-time application of source code. DirectX technology will help to construct the next generation of computer games and multimedia applications. Its content includes the DirectDraw, DirectSound, DirectPlay, Direct3D and DirectInput parts, and they are respectively mainly used in graphics, sound, etc. The function of Direct3D and OpenGL are approximation, they 
provide uniform access to different graphics accelerator card. Direct3D was adopted to realize access to hardware acceleration video with nothing to do with the equipment method. Immediate mode and retained mode, encapsulates the immediate mode, are two alternative ways. The former is suitable for the general 3D application development, while the latter is suitable for quick developers. Direct3D device is Direct3D rendering component, the equivalent of a state machine, packaging and store the render state. Only through the COM interface which is provide by Direct3D, can C++ programs manipulate and use render state and lighting equipment of Direct3D.

\subsection{Virtual reality modeling language VRML}

VRML (virtual reality modeling language) is a kind of 3D exchange format, defining the most common concepts in the $3 \mathrm{D}$ visualization, such as the objects move, rotate, viewpoints, light, material properties, texture mapping, animation, fog, and nested structure, etc. VRML is a descriptive language essentially, the work of drawing the scene entirely by the corresponding browser or plug-in. It uses a standard format to describe the 3D environment, and embedded in Web pages, through plug-ins to provide viewpoints including transformation, flight simulation, control, speed, etc. VRML, as same as HTML, ASCII text format is used to describe and link the world, and it also help to guarantee on a variety of platforms and general, but also reduces the amount of data, which can be achieved on the low bandwidth network. But works of VRML are still staying in a display file stage, lacking of action and interaction.

\subsection{Java3D}

Java language is a network application oriented computer language of SUN Company. Java3D is developed on the basis of Java, OpenGL and VRML. Java language is the extension in the field of 3D graphics, and its essence is a set of API function set. When to use Java3D to write applications, only need to follow the object-oriented thought in appropriate positions for the program corresponding to objects, can quickly write 3D multimedia applications. The API of Java3D contains almost basic classes and interfaces to write JAVA interactive 3D for the application.

\subsection{Interactive Data Language}

Interactive Data Language (IDL) is the product of RSI. It combines visual, interactive analysis, large commercial development at an organic whole, to provide users with perfect, flexible and effective three-dimensional development environment. IDL oriented matrix, grammar are simple, and have strong ability of graphic image processing. It is an ideal tool for the 3D data visualization analysis and application development. The main features of IDL include: the collection of advanced image processing and object oriented programming, and it's a graphics technology which implements interactive 2D and 3D, it also provides cross-platform GUI toolkit, and a variety of program connection tool, it can connect with ODBC compliant database; it is completely open for matrix and has the ability of dealing with large-scale data, and it can handle a variety of formats multiple types of data; what's more, it adopts OpenGL technology and supports OpenGL software or hardware acceleration; With image processing software package, it provides scientific computing model and visual data analysis solutions, etc.

\section{Summary}

The development of 3D visualization, shorten the gap between real world and virtual world of computer, and widened people's horizons. The development of 3D visualization not only make people more clearly understanding the world, but also provide a good guidance for people. And because of virtual reality technology, which is emerging now, can fully reflect the three-dimensional shape, it greatly strengthened the ability of human-computer interaction, and also provided a new train of thought for the study of the 3D visualization of geological body. Virtual Reality modeling language (VRML) is appeared as the interface of Virtual Reality technology, and WWW, it makes many built-in VRML browser can view Virtual objects using VRML programming implementation directly. The fans of VR can write their own procedure which reflect three-dimensional world and experience of realistic three-dimensional world. Therefore, the future of virtual reality will be an important direction and hotspot of the three dimensional visualization technology. 


\section{References}

[1] Javidi, B., Okano, F., \& Son, J. Y. (2009). Three-Dimensional Imaging, Visualization, and Display. Springer.

[2] Downing, E., Hesselink, L., Ralston, J., \& Macfarlane, R. (1996). A three-color, solid-state, three-dimensional display. Science, 273(5279), 1185.

[3] Fenster, A., Downey, D. B., \& Cardinal, H. N. (2001). Three-dimensional ultrasound imaging. Physics in medicine and biology, 46(5), R67.

[4] Javidi, B., \& Okano, F. (Eds.). (2002). Three-dimensional television, video, and display technologies. Springer Science \& Business Media. 